\title{
Didáctica de la lengua y la literatura: entre la intervención y la investigación
}

\author{
Gustavo Bombini \\ Universidad de Buenos Aires y Universidad Nacional de San Martín
}

(Texto recibido el 2 de diciembre de 2018; aceptado el 2 de diciembre de 2018; versión final el 25 de diciembre de 2018)

DOI: https://doi.org/10.5565/rev/jt13.784

Resumen: El propósito de este artículo es indagar acerca de la didáctica de la lengua y la literatura como campo de investigación diferenciado al que caracterizamos como regido no solo por los protocolos habituales y previsibles de cualquier campo de investigación en términos de formas de institucionalización y de legitimación sino también por otras formas específicas de institucionalización y legitimación atendiendo al hecho de que partimos de definir a la didáctica de la lengua y la literatura como una disciplina de intervención, lo cual nos lleva necesariamente a reflexionar sobre las relaciones entre producción de conocimiento en la academia y producción de conocimiento en el campo escolar. Se presentan antecedentes de investigaciones desde el campo de la sociolingüística, el campo de las narrativas y de la etnografía educativa como referencias para la construcción de líneas de investigación en didáctica de la lengua y la literatura.

Palabras clave: didáctica de la lengua y la literatura, investigación, formación docente, disciplina de intervención

\begin{abstract}
The purpose of this article is to investigate the didactics of language and literature as a differentiated field of research. We characterize it as governed not only by the usual and foreseeable protocols of any field of research in terms of forms of institutionalization and legitimation, but also by other specific forms of institutionalization and legitimation based on the fact that we start to define the didactics of language and literature as an intervention discipline. This necessarily leads us to reflect on the relationships between knowledge production in the academy and knowledge production in the school field. We present a background of research from the field of sociolinguistics, the field of narrative and educational ethnography as references for the construction of research trends in language and literature didactics.
\end{abstract}

Keywords: didactics of language and literature, research, teacher training, discipline of intervention

Resum: El propòsit d'aquest article és indagar sobre la didàctica de la llengua i la literatura com a camp d'investigació diferenciat al qual caracteritzem com a regit no només pels protocols habituals i previsibles de qualsevol camp d'investigació en termes de formes d'institucionalització i de legitimació sinó també per altres formes específiques d'institucionalització i legitimació atenent al fet que partim de definir didàctica de la llengua i la literatura com una disciplina d'intervenció, la qual cosa ens porta necessàriament a reflexionar sobre les relacions entre producció de coneixement a l'acadèmia i producció de coneixement en el camp escolar. Es presenten antecedents 
d'investigacions des del camp de la sociolingüística, el camp de les narratives i de l'etnografia educativa com a referències per a la construcció de línies de recerca en didàctica de la llengua $i$ la literatura.

Paraules clau: didàctica de la llengua i la literatura, investigació, formació docent,disciplina d'intervenció

Résumé : Le but de cet article est d'examiner la didactique de la langue et de la littérature en tant que domaine de recherche différencié que nous caractérisons comme étant régi non seulement par les protocoles habituels et prévisibles de tout domaine de recherche en termes de formes d'institutionnalisation et de légitimation, mais également par d'autres formes spécifiques d'institutionnalisation et de légitimation fondées sur le fait que nous définissons didactique du langage et de la littérature comme une discipline d'intervention, ce qui nous amène nécessairement à réfléchir aux relations entre la production de connaissances dans l'académie et la production de connaissances dans le domaine scolaire. Nous présentons des travaux de recherche dans le domaine de la sociolinguistique, de l'ethnographie narrative et de l'ethnographie éducative comme références pour la construction de lignes de recherche en didactique du langage et de la littérature.

Mots-clés : didactique du langage et de la littérature, recherche, formation des enseignants, discipline d'intervention

\section{La didáctica como disciplina de intervención}

El propósito de este artículo es indagar acerca de la didáctica de la lengua y la literatura como campo de investigación diferenciado al que caracterizamos como regido no solo por los protocolos habituales y previsibles de cualquier campo de investigación en términos de formas de institucionalización y de legitimación sino también por otras formas específicas de institucionalización y legitimación atendiendo al hecho de que partimos de definir a la didáctica de la lengua y la literatura como una disciplina de intervención, lo cual nos lleva necesariamente a reflexionar sobre las relaciones entre producción de conocimiento en la academia y producción de conocimiento en el campo escolar.

En este sentido, hemos caracterizado en trabajos anteriores (Bombini, 2015) a la didáctica como disciplina de intervención apuntando al hecho de que la producción de saberes en el campo de la didáctica está directamente vinculada con las prácticas educativas. Es así que esta caracterización de disciplina de intervención atribuida a la didáctica se presenta como un buen primer inicio de acercamiento al campo en tanto su involucramiento directo en términos propositivos y prácticos con el campo de la enseñanza en distintos niveles y modalidades del sistema educativo como así también con el campo de la formación inicial y continua de profesores. La didáctica de la lengua y la literatura, asociada en la mayoría de los 
programas de formación, a lo que se suele denominar "prácticas de la enseñanza", entendidas como las clases de ensayo que marcan la transición entre ser estudiantes y convertirse en profesor, reconoce una vocación de intervención directa en un momento estratégico como son los tramos de finalización de los procesos de formación previstos en los planes de estudio de profesorados de lengua y literatura.

Esta fuerte presencia de la dimensión de las prácticas viene a cuestionar asimismo el propio estatuto de saber de la didáctica en tanto no se trata de una disciplina exclusivamente teórica en consonancia con las matrices de saber más consolidadas en la formación académica, no se reduce a una forma de reproducción de tradiciones de conocimientos legitimados, sino que se configura como una instancia de producción de conocimiento asociada a la práctica, a lo territorial, al trabajo desarrollado en diversidad de contextos e instituciones en los que intervienen sujetos diversos en términos de formaciones, para el caso de los docentes y con trayectorias diferenciadas en el caso de los estudiantes.

Es así que este carácter de disciplina de intervención propicia entonces la participación de diversos actores a través de distintas situaciones de producción en este campo de intervención: formadores (profesores de secundaria), formadores de formadores (profesores de nivel terciario y de universidad, profesores que trabajan en la formación continua), estudiantes, especialistas e investigadores del área; y aquí, la pregunta que se impone es sobre el grado de involucramiento de cada clase de actor interviniendo en relación con la producción de conocimiento y los modos en que los protocolos de la investigación en didáctica de la lengua y la literatura contemplarían la participación de estos múltiples actores en el proceso investigativo.

Definirse como una disciplina de intervención habría de reconocer algún impacto en los modos de hacer investigación a partir de la inclusión del trabajo colectivo en relación con las tareas de enseñanza y de formación. Se trataría de un tipo de investigación atenta a las acciones de intervención que desafía a construir modos de producción nuevos y originales.

Si este trabajo construido entre las disciplinas específicas (la lengua, la literatura) y las prácticas educativas perfila a la didáctica de la lengua y la literatura como una disciplina de carácter interdisciplinario (lo que no es objeto de consideración en este trabajo), por su parte, este modo de construir conocimiento de cara a la práctica, a las intervenciones de enseñanza y de formación y esta participación de multiplicidad de actores en juego invita a repensar los modos de producción de investigación en el campo. Si en la investigación académica usual el investigador autónomo o el investigador que oficia de director y los miembros de equipo son 
los productores de conocimiento, autores reconocidos institucionalmente de las producciones alcanzadas, para el caso de la investigación didáctica -al menos en la versión que nos interesa sostener aquí- tiene también como miembros activos a los propios actores de la práctica.

\section{Antecedentes posibles}

En el proceso de construcción de líneas de investigación en didáctica de la lengua y la literatura se ha atendido a diversos antecedentes acaso no específicos referidos a la investigación lingüística y educativa pero cuyo interés podrá ser reconocido en desarrollos ya realizados y otros en ciernes que muestran la productividad de este campo cuyo proceso más potente de configuración data de los últimos treinta años.

El carácter de disciplina de intervención de la didáctica de la lengua y la literatura nos lleva a recuperar aquellos paradigmas que de algún modo suponen el trabajo posible en territorio y que reconocen el interés de la participación de sujetos y comunidades en los procesos investigativos.

Mucho se ha hablado de metodologías mencionadas, en general, como "investigación participativa" (Sirvent, 1999) y quizá algunos aspectos de estas tradiciones de investigación, ya desarrolladas en el campo de la educación popular y en la educación de adultos, constituirían un aporte novedoso para el campo de la didáctica de la lengua y la literatura.

En esta ocasión prefiero detenerme en algunas lecturas y líneas de trabajo que nos permitieron avanzar en la producción de conocimientos pedagógico-didácticos que se fueron gestando en el propio territorio de la intervención en la formación de profesores y que brindan fundamentos y preguntas enriquecedoras para esta discusión en torno a la investigación en didáctica de la lengua y la literatura.

Desde el propio campo disciplinario, interesa recuperar las reflexiones que hace algunas décadas realizaba Michel Stubbs en su trabajo ya clásico sobre la interacción en el aula desde la perspectiva sociolingüística (Stubbs, 1984) en el que destaca -acaso de manera paradójica- las limitaciones de los investigadores del campo de la sociolingüística para realizar observaciones en el aula, frente a la riqueza y diversidad de los modos en que se producen los verdaderos intercambios en el aula. Según Stubbs existiría un lenguaje "auténtico" que difícilmente se despliega frente a la artificiosidad de la presencia del investigador, a la vez que estas investigaciones parten de categorías descriptivas que no son de naturaleza sociolingüística. 
Stubbs va a ir más allá para criticar las nociones de código restringido y código elaborado de Basil Bernstein, de gran influencia en las consideraciones entre lenguaje y educación y va a sostener que "la limitación de la obra de Bernstein, desde el punto de vista de su capacidad para hacer afirmaciones precisas y comprobables, es la casi total falta de ejemplificación lingüística de sus teorías (p. 57)". Asimismo, va a sostener que "si queremos saber cómo se comporta la gente en las aulas, no cabe duda de que tendremos que observarla en las aulas" (p.57). Y agrega -con ironía-: "Si la llevamos al laboratorio psicológico de la universidad local, entonces podremos descubrir la forma en que se comportan allí." A partir de estos argumentos, en apariencia obvios, Stubbs va a proponer realizar "observaciones y análisis del auténtico comportamiento diario de profesores y alumnos en clase" (p. 96). Si bien los profesores no están -según Stubbs- familiarizados con las investigaciones porque estas no se relacionan de manera clara con las cosas que ocurren en "la jungla del aula", ni podrán realizar investigaciones en laboratorio si "son perfectamente capaces de observar lo que ocurre en las aulas" (p. 96) y respecto de la participación de estos profesores en la investigación es contundente: "la investigación que se realiza en las aulas comprende puntos de vista que cualquier estudiante o cualquier profesor con experiencia podrá verificar, corregir o refutar siempre que esté trabajando en un aula" (p. 96). Y por fin ratifica su posición: "Las investigaciones sobre niños y aulas suelen realizarlas los "extraños"; pero en, último término, sólo los participantes en una situación tendrán acceso total a todos sus aspectos relevantes” (p. 96) y avala su posición en una frase de Hymes: "la etnografía de una situación no debe ser descrita por quien no participa en ella" (p. 96). Pero además agrega razones de índole cuantitativa. "Sencillamente, si el estudio del lenguaje del aula debe depender de investigadores externos a él, nunca se llevará a cabo en la mayoría de las aulas” (p. 96).

Está claro que las preocupaciones de Sttubs se centran en la mirada sociolingüística sobre la interacción en el aula, pero podríamos recuperarlas para las consideraciones en torno a la didáctica de la lengua y la literatura como disciplina de intervención y como campo de investigación y recuperar esta idea de la necesidad de abordar las prácticas de enseñanza en el aula de la mano de quienes están adentro de las aulas. En este sentido, las intervenciones de los investigadores en didáctica de la lengua y la literatura, ajenos al aula, no acabarían por ofrecer una mirada acabada sobre la complejidad de las prácticas, ni estarían en condiciones de producir cierto tipo de conocimiento emergente de la práctica misma; invisibilizado como tal, deslegitimado, si no fuera recuperado desde un cierto tipo de investigación a realizar desde la didáctica de la lengua y la literatura. 
Otro aporte que ayuda a pensar la cuestión de las relaciones entre prácticas de intervención e investigación en didáctica de la lengua y la literatura proviene de aquellas experiencias y corrientes pedagógicas que sostienen la idea de los profesores como investigadores de sus propias prácticas. Las experiencias e investigaciones de Marilyn Cohran-Smith y Susan Lytle incluidas en el libro Dentro/fuera: Enseñantes que investigan (publicado en inglés de 1993 y por primera vez en español de 2002) parten de la necesidad de poner en discusión el supuesto ampliamente aceptado de que el conocimiento sobre la enseñanza se produce en el ámbito de la investigación universitaria. Las autoras van a sostener la tesis de que la investigación a cargo de profesores "representa una forma especial de conocimiento sobre la enseñanza y el aprendizaje que puede transformar (no solo añadirse) a lo que conocemos en el campo" (p. 133). Sostienen además que el desarrollo de este tipo de investigación vendrá asimismo a cuestionar los supuestos "sobre las relaciones entre la teoría y la práctica, entre la escuela y las universidades, y entre la investigación y la reforma” (p. 133).

Acaso el desarrollo más interesante que realizan Cohran-Smith y Lytle en relación con el tema de este artículo es el planteo que hacen las autoras respecto de la necesidad de rescatar el conocimiento sobre la enseñanza producido por profesores reconociendo en él una especificidad epistemológica que lo diferencia de la investigación universitaria. Las autoras sostienen que "la investigación hecha por docentes (...) hace accesible parte de la pericia docente y aporta a las universidades y a las comunidades educativas perspectivas únicas sobre la enseñanza y el aprendizaje" (p. 139), es decir, con esta afirmación se invierte la relación arriba-abajo que va de universidad a escuela, para plantear que las comunidades educativas son también espacios de producción de cierto conocimiento específico.

Asimismo, las autoras se detienen en dos aspectos fundamentales que son la institucionalización de la investigación hecha por enseñantes y los parámetros de rigor metodológico que se ponen en juego en este tipo de investigación, diferente a la investigación universitaria. Con respecto a la institucionalización destacan los trabajos de tesinas o de licenciaturas que llevan adelante enseñantes de la educación obligatoria cuando asumen estudios de posgraduación; otros enseñantes forman parte de proyectos universitarios de tipo colaborativo y por fin, otros, se asocian entre sí formando grupos de investigación entre docentes colegas. Depende de las estructuras de la burocracia ministerial el hecho de que eventualmente alguno de estos grupos pudiera recibir algún tipo de subsidio económico para el desarrollo de sus investigaciones. Señalan también, como una variable obstaculizadora del 
trabajo de investigación, el exceso de horas de clase, la rigidez de los horarios, la escasez de foros, jornadas y otros eventos locales o nacionales en las que se divulguen y se publiquen las producciones de los profesores que investigan.

Respecto de los estándares de rigor metodológico las autoras reconocen que las investigaciones de docentes parten de la experiencia cotidiana y que esto no constituye un aspecto trivial, sino que constituye el núcleo reflexivo más importante acerca de la propia práctica referenciado en los contextos particulares en las que ellas se desarrollan. No se trata de preguntas meramente instrumentales referidas a recursos o a técnicas didácticas, sino que se trata de preguntas más generales que hacen a una hermenéutica de los hechos escolares, de las situaciones de enseñanza y aprendizaje donde se hallan implícitas teorías y reflexiones pedagógicas de gran generalidad.

Por fin recapitulando las distintas experiencias, las autoras van a sostener la existencia de "un amplio abanico de textos" entre las producciones de comunidades de profesores. De este modo, diarios, investigaciones orales, estudios de aula y de centro, notas de campo sobre observaciones de clases, registros escritos de discusiones entre profesores, así como también trabajos de investigación conceptual al modo de ensayos teórico-filosóficos o de análisis de ideas, conforman un corpus productivo desde el cual las comunidades de profesores pueden producir conocimiento acerca de la práctica. Escritos en algunos de estos géneros se publican en Dentro/fuera. Enseñantes que investigan algunas producciones a cargo de profesores que han participado en grupos de investigación.

Otro aporte a estas consideraciones acerca de la investigación en didáctica de la lengua y la literatura, inscripto en las líneas de investigación narrativa, es el que realizan Michael Connelly y Jean Clandinin en el artículo "Relatos de experiencia e investigación narrativa". La noción de "narrativa” propia de esta línea asume dos sentidos, por un lado, es el fenómeno que se investiga y a la vez, narrativa es el método de investigación que se elige. La educación se considera parte de la actividad humana entendida ésta como una actividad eminentemente narrativa: los humanos contamos historias, relatamos vidas. La experiencia vivida cobra relevancia en la investigación educativa de orientación cualitativa entre cuyas líneas se encuentran las investigaciones sobre el currículo, más cercanas a la práctica escolar. Relatos de profesores o relatos sobre profesores se constituyen como parte del dispositivo metodológico para el desarrollo de investigaciones realizadas por profesores en los que la voz de cada uno tiene relevancia para el avance de la investigación; los datos empíricos son clave 
en el desarrollo de este tipo de investigaciones, datos que serán sometidos al trabajo de interpretación con una cierta asociación al trabajo de la crítica literaria.

También en este caso, los autores proponen géneros de escritura y de documentación propios del trabajo docente que formarían parte de la investigación como son las notas de campo de la experiencia compartida, anotaciones en diarios, transcripciones de entrevistas, observaciones, cartas, documentos (programaciones de clases, entre otras), fotografías, registros fílmicos, entre otros.

El trabajo de investigación biográfico-narrativa es propuesto por los académicos españoles Antonio Bolívar, Jesús Domingo, Manuel Fernández Cruz en su libro La investigación biográfico-narrativa en educación (Bolívar et al., 2001) inscriben la relevancia de este tipo de investigación en el giro hacia la perspectiva interpretativa que se produce en las ciencias sociales en los años setenta y destacan el hecho de que desde esta perspectiva se rescatan las voces de quienes ejercen el oficio de enseñar. Distintos aspectos complejos, singulares, emotivos, son captados en su detalle y riqueza por el relato que recupera las intenciones humanas de los hechos de la experiencia.

También los autores destacan el carácter interdisciplinar en la investigación narrativa donde se cruzan diversas ciencias humanas y sociales como la teoría lingüística y literaria, la antropología social, la etnografía, la sociología, la historia oral, la retórica, la filosofía hermenéutica, entre otros campos.

\section{Nuestra investigación}

Como parte de la reflexión acerca de los modos de hacer investigación en didáctica de la lengua y la literatura y en relación con la formación de profesores queremos dar cuenta de nuestra propia investigación realizada por un equipo que se dedica a la formación de profesores en Letras en la Universidad de Buenos Ares.

Dentro del trabajo de la Cátedra de Didáctica Especial y Prácticas de la Enseñanza se ha promovido la diversificación de los textos posibles para la construcción de la tarea de formación de profesores. Esto ha llevado a revisar los formatos habitualmente usados como son la planificación y las llamadas “Guías de Observación”. En este sentido es la etnografía y sus desarrollos de escritura la línea metodológica que ha proporcionado más aportes y en diferentes trayectos se ha propuesto la escritura de registros, notas de campo, diarios y se ha recurrido a otros géneros de creación como los "autorregistros" para dar cuenta de la práctica en proceso y los "guiones conjeturales" (Bombini, 2004) en las instancias de planificación. 
Asimismo, se ha avanzado en la construcción de categorías descriptivas y de análisis que permiten sistematizar el conocimiento de estos géneros de escritura de la práctica y observar y evaluar el impacto que su escritura tiene en la producción de la propia práctica. Se ha trabajado específicamente en el análisis de las formas y las funciones de los llamados "autorregistros" o "autoinformes" y el "guion conjetural" como textos de la práctica utilizados por los practicantes y se ha analizado su incidencia en la tarea de planificación y de análisis en la construcción de la propia práctica de enseñanza de la lengua y la literatura, así como también en la productividad de la escritura de ficción en los procesos de construcción del conocimiento. En términos generales podemos decir que ambos tipos de textos propician una posición reflexiva en relación con la práctica y con sus procesos de construcción. Asimismo, permiten cuestionar los presupuestos de previsibilidad y control total y estricto de la práctica, características que se presentan naturalizadas en la planificación tradicional. Se cuestiona a partir de estos géneros, a su vez, el carácter fuertemente normativo y prescriptivo de los textos de la práctica en tanto que estas características se presentan como obturantes de su desarrollo. De este modo los alumnos practicantes a partir de una tarea de ida y vuelta de escritos van desterrando formas estereotipadas de concebir la práctica y la propia tarea de reflexión en la escritura permite reconocer sus aspectos problemáticos, momentos interesantes en su desarrollo, aspectos vinculados a la naturaleza de las decisiones tomadas y sus implicancias en lo específicamente didáctico.

En cuanto a los géneros y sus características, se ha visto la productividad que supone, en tanto posicionamiento responsable y procesos de construcción de identidad frente a la práctica, el uso de la primera persona tanto en el autoinforme como en el guion conjetural. Asimismo, también resulta significativo el juego de inclusión de voces ajenas que son las voces propias de la situación de aula y que se convierten en voces que se traman en fuerte operación de construcción de sentido con la voz del "autor" de la práctica y se presentan a su vez como voces que dicen sobre la práctica, sobre los modos de su construcción, sobre sus propias experiencias socioculturales.

Sabemos que, desde distintos campos y paradigmas de la investigación en las ciencias sociales, en las ciencias del lenguaje y en las disciplinas relacionadas con la formación docente se viene reconociendo la importancia de las prácticas de escritura en la producción de conocimiento y en los procesos de formación. Por una parte, existe un diagnóstico generalizado referido a las escasas competencias de lectura y escritura de los estudiantes de nivel superior que da lugar a desarrollos propositivos a nivel individual e institucional acerca 
de cómo abordar estos déficits. En este sentido, aquellas corrientes enroladas en lo que se ha dado a llamar "alfabetización académica" postulan la necesidad de establecer dispositivos para el desarrollo de las prácticas de lectura y escritura en distintos espacios y tiempos de la formación de los estudiantes (Carlino, 2005).

Pero más allá de los géneros académicos de la formación superior en general (exámenes escritos, monografías) esta propuesta formativa y la correspondiente investigación que aquí comentamos parten del reconocimiento de otros géneros propios del recorrido particular que supone la formación de profesores: se trata de géneros de escritura ligados a acciones pedagógicas, en el sentido que las anticipan o las recuperan como objeto de construcción y de reflexión. Esta ligazón entre acciones didácticas y géneros de la práctica propicia la necesidad de producir otros modos de construcción de la relación con el conocimiento en tanto el escenario de la práctica propone el desafío de la recontextualización de los saberes y su escritura y la exploración de géneros específicos.

Distintas investigaciones tanto históricas (Diker, 2006, Alliaud, 1999) como las ya referidas vinculadas con procesos de la formación docente inicial y continua (Connelly y Clandinin, 1995, Cochran-Smith y Lytle, 2002) han problematizado la cuestión de la escritura y su relación con la profesión docente y han demostrado que las posibilidades de su desarrollo no se reducen a los reconocibles textos de la burocracia escolar -especialmente la planificación- sino que tanto en la historia como en experiencias específicas de trayectorias docentes o de grupos las posibilidades genéricas se amplían, se diversifican y muestran variadas posibilidades creativas.

En este sentido, desde el espacio de formación de la Cátedra Didáctica Especial y Prácticas de la Enseñanza en Letras -a la que pertenece la totalidad de los miembros de este equipo de investigación- venimos indagando acerca de diversos géneros, a los que hemos llamado "géneros de la práctica" entre los que se destacan los "guiones conjeturales" (Bombini, 2001 y 2006 y Bombini, Labeur, 2013) y los autorregistros (Bombini, 2012 y Bombini, Labeur, 2013), así como también géneros de la práctica en uso en el momento de la formación inicial como notas de campo y breves textos ensayísticos generados a partir de la observación de clases de lengua y literatura y también (en el marco de una investigación en curso) el desarrollo de ciertos géneros de ficción con un componente argumentativo (diálogos platónicos, cielitos de la tradición gauchesca, escenas teatrales, guiones de historieta) como posibilidad de desarrollar nuevas estrategias retóricas para la apropiación y evaluación de la saber didáctico específico. ${ }^{\mathrm{i}}$ 
Todas estas incursiones a través de géneros posibles de escritura en la formación docente reconocen también nuevas formas de diversificación en el contexto de las transformaciones que la multimodalidad en el marco de las TIC viene demostrando como modos diferentes de construir relaciones de apropiación con la cultura escrita (Jewitt, 2015).

Estas relaciones que incluyen vínculos con otras prácticas culturales (visuales, audiovisuales, musicales, entre otras) y con las TIC reconocen una presencia significativa en las aulas de la escuela secundaria - destino laboral de los profesores en formación- a partir de las propias experiencias culturales de los estudiantes y/o del impacto de las políticas educativas de inclusión digital (Programa Conectar-Igualdad), pero aún no han sido suficientemente exploradas en los procesos de la formación docente y en la investigación referida a ella. La pregunta que se impone es acerca del modo en que las prácticas de lectura y escritura en su entrecruzamiento con la multimodalidad tienen lugar en la formación docente.

Las tareas de formación de profesores en lengua y literatura realizadas en el marco de la Cátedra de Didáctica Especial y Prácticas de la Enseñanza en Letras, así como la desarrollada en otras cátedras del país y del extranjero, cuando se convierten en objeto de investigación, permiten avanzar en la construcción de conocimiento específico en este terreno. Frente a la visión simplificadora que reduce el "hacer las prácticas" a la tarea de lograr cierta "enseñabilidad exitosa" de contenidos lingüísticos y literarios (como tarea naturalizada de la didáctica de la lengua y la literatura) se impone la complejidad de la práctica en tanto ésta se desarrolla en distintos escenarios escolares, en tanto participan de ella diversos sujetos, y en tanto los dispositivos materiales y tecnológicos en los que se apoya la tarea de enseñanza se diversifican y se enriquecen en el contexto de una mirada ampliada de la enseñanza como práctica cultural compleja.

De este modo, la producción de variadas consignas de trabajo elaboradas e implementadas tanto en las clases teóricas y prácticas como durante el desarrollo de las prácticas docentes que invitan al establecimiento de relaciones entre textos escritos y diversos objetos y modalidades culturales ofrecerán ricas posibilidades de análisis y de reflexión sobre el modo en que se construyen las prácticas y la reflexión sobre ellas y el propio rol docente.

$\mathrm{Si}$-como hemos comprobado- escribir "guiones conjeturales" ii en tanto textos de anticipación era "inventar" los modos en que una determinada escena de aula se produciría y avanzar en la lectura de textos ficcionales (de la tradición literaria o producidos por los propios compañeros) que representen situaciones relacionadas con la enseñanza de la lengua y la literatura, ponía en juego un modo particular y productivo de posicionarse los sujetos de la 
práctica en relación con la práctica misma, nos proponemos en una nueva etapa del proyecto atravesar la experiencia de trabajo con la multimodalidad en tanto permitirá desarrollar nuevos dispositivos y estrategias para la formación docente y para la enseñanza de la lengua y la literatura.

Para el desarrollo de este proyecto se ha recurrido a un modelo de investigación cualitativa (Vasilachis, 1993) cuya productividad en el campo de la enseñanza de la lengua y la literatura y en el análisis de las prácticas sociales de lectura y escritura se viene observando ya sea en investigaciones de corte más cultural (Petit, 1999 y 2001), como en las que trabajan de manera específica en escenarios escolares (Rockwell, 1995, 2009). Asimismo, y recuperando líneas de investigación del equipo a cargo que se han centrado en el análisis de experiencias de enseñanza y formación a partir de la producción de diversidad de textos escritos (Bombini, 2003, 2004 y 2012), se propone el armado de un corpus de textos y objetos culturales que actualmente se halla en proceso de construcción y que podría sistematizarse de la siguiente manera:

- Trabajos escritos, presentados con diseños y resoluciones tipográficos que resulten significativos para su interpretación.

- Guiones para la producción de narrativas ilustradas e historietas que den cuenta de perspectivas y prácticas de formación y enseñanza desarrollados por los alumnos para la preparación de sus clases.

- Testimonios audiovisuales (storyboard, bocetos, ilustraciones, fotos, películas o fragmentos de películas, breves filmaciones de entrevistas a referentes del campo, a docentes, a alumnos, y registros de clases) seleccionados y disponibles en el espacio de la cátedra en el campus virtual o producidos por los alumnos.

-Producciones didácticas multimodales para su utilización en las prácticas docentes.

Se trata de un corpus inicial que está sujeto a posibles diversificaciones a medida que se avance en las prácticas de formación en las que distintas situaciones de enseñanza den lugar a la necesidad de recurrir a otros géneros propios de la multimodalidad.

\section{Reflexiones finales}

El posicionamiento y los ejemplos de investigaciones posibles en el campo de la didáctica de la lengua y la literatura están intentando mostrar cierto sentido metodológico, pero a la vez político, del trabajo en este campo. Decimos que la didáctica de la lengua y la literatura se caracteriza por ser una disciplina que se define por su capacidad de intervenir en 
el campo específico de la enseñanza y de la formación docente, a la vez que en otros espacios del más allá de la escuela. Y esta "vocación de intervención” genera a la vez la necesidad de establecer los modos en que se producirán las articulaciones entre el campo académico y el campo escolar en dos dimensiones: por una lado en relación con las tareas propias de la formación docente ("hacer las prácticas", en tanto el desarrollo de prácticas de enseñanza entendidas como una gestión colaborativa entre instituciones formadoras e instituciones de enseñanza) y por otro, en relación con la investigación acerca de la didáctica de la lengua y la literatura. El desafío es metodológico pues se trata de avanzar en la investigación a partir de considerar la posibilidad de participación de diversos sujetos en el proceso investigativo, de modo tal que no sean solo los universitarios los que participen de estas tareas, si no que el profesorado se integre como un actor más, productor de cierta forma de conocimiento experiencial diferenciado del conocimiento académico; este conocimiento académico que además se nutre de los insumos que vienen del campo de la teoría se pone en diálogo con el conocimiento que se configura a partir de la práctica lo que invita a atravesar otros desafíos epistemológicos y metodológicos propios de esta forma de producción de investigación que aquí estamos caracterizando.

La posibilidad de que se desdibujaran estas articulaciones entre la academia y la práctica, entre el mundo universitario y el mundo escolar, entre producción de conocimiento desde la teoría, y producción de conocimiento desde la práctica, pondría en riesgo la impronta intervencionista $\mathrm{y}$, en definitiva, transformadora de la didáctica hacia el campo de la formación docente y hacia el campo de la enseñanza de estas disciplinas. El desarrollo de la didáctica de la lengua y la literatura como campo específico ha dado lugar a la configuración de un nuevo campo de investigación que comienza asumiendo las prácticas usuales y los rituales propios de cualquier campo pero que no debe dejar de reconocer que en su singularidad habrá de mostrar (en el sentido de que no habrá de inhibir) su complejidad, la de su propia lógica, y la de sus razones políticas, su intervención en los campos de la enseñanza y de la formación de profesores.

Hemos planteado en trabajos anteriores (Bombini, 2015) que la consolidación del campo de la didáctica de la lengua y la literatura en el campo académico acarrearía el riesgo de asumirse a sí misma y posicionarse como parte de una lógica endogámica, sometida a las reglas del juego del juego académico, pero desatendiendo a las otras reglas, las de otro juego que es el juego de la tarea en las instituciones escolares. Advertimos entonces acerca del riesgo de la "fuga academicista" de la didáctica donde su propio crecimiento sería el motivo 
para que se desvirtúen algunas marcas de origen. Ligadas a los trayectos formativos de los profesorados (en general universitarios) las cátedras de didáctica y prácticas de la enseñanza vinieron a dar cuenta de una realidad cuantitativa y políticamente significativa, la de la cantidad de graduados universitarios, formados en el oficio de profesor que ocuparan espacios en el sistema de enseñanza público, en el subsistema de la formación docente y, como dijimos, también en otros espacios del más allá de la escuela donde la lengua y la literatura son objeto de algún interés o de alguna producción.

Esta marca de origen, ligada a las prácticas de formación, advierte a los investigadores acerca de la necesidad de no distraer el compromiso con esa zona de intersección con el campo de la enseñanza y con el campo de la formación como prácticas sociales desde donde se proyectan los nuevos aportes propios de una didáctica de la lengua y la literatura que no se presenta como normativa, ni como prescriptiva sino como un campo activo de intervención y de producción de conocimiento pedagógico-didáctico crítico.

\section{Referencias}

Alliaud, A. (1999). Las autobiografías escolares como métodos e instrumentos de indagación y transformación de la docencia. Revista Ensayo y Error, 8 (16/17), 51-72.

Bolívar, A., Domingo, J., y Fernández Cruz, M. (2001). La investigación biográfico-narrativa en educación: Enfoque y metodología. Madrid: La Muralla.

Bombini, G. (2003). Poner las prácticas en texto: Un modo de saber acerca de la capacitación. Ficha de cátedra. Buenos Aires: CEPA.

Bombini, G. (2004). Prácticas docentes y escritura: hipótesis y experiencias en torno a una relación productiva. Ponencia presentada en el Congreso Nacional de Prácticas Docentes, Universidad Nacional de Córdoba, Córdoba.

Bombini, G. (coord.) (2012). Escribir la metamorfosis: Escritura y formación docente. Buenos Aires: El hacedor.

Bombini, G. (2015). Textos retocados: Sobre lengua, literatura y enseñanza. Buenos Aires: El hacedor.

Bombini, G., y Labeur, P. (2013). Escritura en la formación docente: los géneros de la práctica. Revista Enunciación, 18 (1), 19-29. DOI: https://doi.org/10.14483/22486798.5715

Carlino, P. (2005). Escribir, leer y aprender en la universidad: Una introducción a la alfabetización académica. Buenos Aires: Fondo de Cultura Económica. 
Cohran-Smith, M., y Lytle, S.L. (2002). Dentro/Fuera: Enseñantes que investigan. Madrid: Akal.

Connelly, M., y Clandinin, J. (1995). Relatos de Experiencia e Investigación Narrativa. En J. Larrosa, R. Arnaus, V.R. Ferrer, N. Pérez de Lara, M. Connelly, J. Clandinin, M. Greene (eds.), Déjame que te cuente: Ensayos sobre narrativa y educación (pp. 1159). Barcelona: Laertes.

Diker, G. (2006). El maestro como autor en la prensa pedagógica argentina (1958-1930) (Tesis de doctorado inédito). Universidad del Valle, Cali, Colombia.

Jewitt, C. (2015). Multimodalidad, lectura y escritura para el siglo XXI. Lulú Coquette. Revista de Didáctica de la Lengua y la Literatura, 7, 23-45.

Petit, M. (1999). Nuevos acercamientos a los jóvenes y la lectura. México: Fondo de Cultura Económica

Petit, M. (2001). Lecturas: Del espacio íntimo al espacio público. México: Fondo de Cultura Económica

Rockwell, E. (1995). En torno al texto: tradiciones docentes y prácticas cotidianas. En E. Rockwell (ed.), La escuela cotidiana (pp. 198-222). México: Fondo de Cultura Económica.

Rockwell, E. (2009). La experiencia etnográfica: Historia y cultura en los procesos educativos. Buenos Aires: Paidós.

Sirvent, M.T. (1999). Cultura popular y participación social. Buenos Aires: Miño y Dávila.

Stubbs, M. (1984). Lenguaje y escuela. Análisis sociolingüístico de la enseñanza. Madrid: Cincel-Kapelusz.

Vasilachis, I. (1993). Métodos cualitativos I. Los problemas teórico epistemológicos. Buenos Aires: CEAL.

${ }^{\text {i }}$ Proyecto financiado UBACyT (2010-2012), Universidad de Buenos Aires - Secretaría de Ciencia y Técnica.
ii Proyecto financiado UBACyT (2006-2009), Universidad de Buenos Aires - Secretaría de Ciencia y Técnica.

Información del autor

Gustavo Bombini es profesor y doctor en Letras. Profesor e investigador en las universidades de Buenos Aires y Nacional de San Martín. Director del Profesorado Universitario en Letras en la Universidad Nacional de San Martín.

Email: gbombini@gmail.com 
Para citar este artículo:

Bombini, G. (2018). Didáctica de la lengua y la literatura: entre la intervención y la investigación. Bellaterra Journal of Teaching \& Learning Language \& Literature, 11(4), 5-20. DOI: https://doi.org/10.5565/rev/jtl3.784 